\title{
SOBRE LA DENEGACIÓN DE RECONOCIMIENTO EN ESPAÑA DE UNA MEDIDA CAUTELAR CONTENIDA EN UN LAUDO ARBITRAL EN MATERIA CONCURSAL DICTADO EN LA FEDERACIÓN RUSA
}

\author{
ON THE DENIAL OF RECOGNITION IN SPAIN OF A \\ PROTECTIVE MEASURE CONTAINED IN AN ARBITRAL \\ AWARD IN BANKRUPTCY ISSUED IN THE RUSSIAN \\ FEDERATION
}

\author{
José LuIS IRIARTE ÁNGEL \\ Catedrático de Derecho Internacional Privado \\ Universidad Pública de Navarra \\ ORCID ID: 0000-0002-2353-5003
}

Recibido: 04.04.2020 / Aceptado: 05.05.2020

DOI: https://doi.org/10.20318/cdt.2020.5652

\begin{abstract}
Resumen: En el artículo se aborda el problema del reconocimiento y ejecución en España de una medida cautelar dictada en Rusia en un procedimiento arbitral de naturaleza concursal. El Tribunal español aplica al caso el Convenio de Nueva York de 10 de junio de 1958 sin realizar ninguna justificación al respecto y como si diese por supuesto que el exequatur de medidas cautelares arbitrales se rige por el mismo. Rechaza la pretensión del demandante por dos motivos, falta de presentación del convenio arbitral y que la decisión recae sobre una materia inarbitrable en nuestro Derecho.

Palabras clave: reconocimiento y ejecución, medida cautelar arbitral extranjera, procedimiento concursal, Convenio de Nueva York de 10 de junio de 1958, convenio arbitral, inarbitrabilidad de la diferencia.
\end{abstract}

Abstract: This article addresses the problem of the recognition and execution in Spain of a protective measure issued in Russia in an arbitration procedure of a bankruptcy nature. The Spanish Court applies the New York Convention of June 10, 1958 to the case without any specific justification as if it were assumed that the exequatur of an arbitration protective measure is governed by it. It rejects the plaintiff's claim for two reasons, failure to submit the arbitration agreement and that the decision falls on a matter that under our legal system can not be submitted to arbitration.

Keywords: recognition and execution, foreign arbitration protective measure, bankruptcy procedure, New York Convention of June 10, 1958, arbitration agreement, non-arbitrability of the dispute.

Sumario: I. Antecedentes de hecho y algunas precisiones al respecto. II. Puntualizaciones en cuanto a la normativa aplicada por el Tribunal. III. Motivos por los que la Sala denegó el reconocimiento. IV. Un problema subyacente: el reconocimiento y ejecución de medidas cautelares adoptadas por árbitros en el extranjero. V. Conclusiones. 


\section{Antecedentes de hecho y algunas precisiones al respecto}

1. El Auto del Tribunal Superior de Justicia de Cataluña (Sala de lo Civil y Penal, Sección $1^{\mathrm{a}}$ ), de 19 de septiembre de $2019^{1}$, tiene su origen en la solicitud de reconocimiento de un laudo arbitral dictado el 19 de marzo de 2019 por el Tribunal de Arbitraje de la Región de Rostov del Don (Federación Rusa). Se trataba de una resolución cautelar dictada en un procedimiento de naturaleza concursal, instado por un banco, en el que se había declarado como insolvente (en quiebra) al demandado, que era una persona física, y se había aprobado un plan de restructuración de las deudas del mismo. El Administrador concursal nombrado por el Tribunal Arbitral pretendía al presentar la demanda el exequatur de la decisión de dicho Tribunal, dictada en el curso del procedimiento de quiebra, por la que se prohibía al demandado enajenar una finca de su propiedad sita en Lloret de Mar, con el fin de que la decisión pudiese ser ejecutada mediante su inscripción en el Registro de la Propiedad competente. El Tribunal Superior de Justicia de Cataluña denegó el exequatur por dos motivos a los que nos referiremos más tarde.

2. Antes de proseguir es necesario hacer referencia a una cuestión, que al parecer no se planteó en este caso pero que puede tener notable relevancia práctica. Me refiero al problema de precisar si el Tribunal de Arbitraje de la Región de Rostov del Don era realmente un órgano arbitral que dictó un auténtico laudo arbitral. En los Estados del espacio postsoviético existen instituciones que aunque reciben el nombre de arbitrales realmente son unos Tribunales estatales, lógicamente las resoluciones que dictan estos órganos no son auténticos laudos arbitrales y por consiguiente no pueden alcanzar eficacia en España a través de las disposiciones procesales y sustantivas previstas para el exequatur de las resoluciones arbitrales sino por medio de la normativa reguladora del reconocimiento y la ejecución de las sentencias judiciales. Así lo dijo el Tribunal Superior de Justicia de Cataluña en un Auto de 7 de enero de $2015^{2}$, en el que, ante la solicitud de exequatur de un laudo dictado por el Tribunal de Arbitraje Económico de Kiev, apreció correctamente que realmente estaba ante una sentencia dictada por un Tribunal Mercantil, que no era una institución ajena a la jurisdicción del Estado sino un órgano judicial especializado en controversias comerciales, y en consecuencia el propio Tribunal Superior de Justicia no era competente para resolver el exequatur ni se podía aplicar la normativa atinente al reconocimiento y ejecución de los laudos. Este problema no se planteó en el caso que ahora nos ocupa, pero tal vez subyacía en él, pues no deja de ser significativo que uno de los motivos por los que no se accedió a la pretensión del demandante fue porque no presentó con la demanda el original o copia auténtica del convenio arbitral. Por otro lado, hay que considerar que posiblemente hubiese sido más fácil obtener la eficacia en España de la resolución rusa si se hubiese planteado el exequatur como el de una decisión judicial en materia concursal.

\section{Puntualizaciones en cuanto a la normativa aplicada por el Tribunal}

3. La parte demandante invocó en apoyo de sus pretensiones los artículos 12.1 de la Ley de la Jurisdicción Voluntaria ${ }^{3}, 54$ de la Ley de Cooperación Jurídica Internacional en Materia Civil ${ }^{4}$, IV del Convenio de Nueva York de 10 de junio de 1958 sobre reconocimiento y ejecución de sentencias arbitrales extranjeras ${ }^{5}$ y 46 de la Ley de Arbitraje ${ }^{6}$. A este respecto hay que hacer algunas precisiones en cuanto a determinadas disposiciones que no se invocaron en la demanda y a la idoneidad de las que sí se citaron.

\footnotetext{
${ }^{1}$ ECLI:ES:TSJCAT:2019:570A. JUR\2019\336067.

2 ECLI:ES:TSJCAT:2015:15A.

${ }^{3}$ BOE de 3 de julio de 2015; rectificación en BOE de 2 de septiembre de 2015.

${ }^{4}$ BOE de 31 de julio de 2015.

${ }^{5}$ BOE de 11 de julio de 1977; rectificación en BOE de 17 de octubre de 1986.

${ }^{6}$ BOE de 26 de diciembre de 2003.
} 
4. El demandante no aludió para amparar su pretensión al Convenio europeo de Ginebra, de 21 de abril de 1961, sobre arbitraje comercial internacional ${ }^{7}$, del que tanto España como Rusia son Estados miembros. Su posición fue correcta, pues de acuerdo con lo dispuesto en el artículo 1.1 del texto convencional el mismo sólo se aplica en los casos en los que las partes tengan su residencia habitual o su domicilio o sede social en Estados contratantes diferentes y en este supuesto parece que ambas partes tenían su domicilio en Rusia. No obstante, aun en la hipótesis de que el Convenio hubiese sido aplicable el resultado final del procedimiento de exequatur no hubiese variado.

5. Tampoco invocó el demandante el Convenio bilateral entre España y la Unión Soviética, de 26 de octubre de 1990, sobre asistencia judicial en materia civil ${ }^{8}$. Una reiterada jurisprudencia ha sostenido que el mencionado Convenio rige en la actualidad el exequatur de sentencias rusas en España; así en el Auto del Tribunal Supremo (Sala de lo Civil) de 9 de febrero de 1999 ' se dijo que "Existente un Convenio entre el Reino de España y la Unión de Repúblicas Socialistas Soviéticas sobre asistencia judicial en materia civil, firmado en Madrid el 26 de octubre de 1990, que entró en vigor el 22 de julio de 1997, habiendo dejado de existir la Unión de Repúblicas Socialistas Soviéticas -las cuales dieron lugar a la aparición de diversos Estados, entre ellos la Federación de Rusia- y siendo el demandado de exequatur un nacional de dicha Federación, ha de pronunciarse esta Sala sobre la aplicabilidad del citado Convenio a la Federación Rusa. Examinada la Convención de Viena de 23 de mayo de 1969 sobre el Derecho de los Tratados y fundamentalmente la Convención de Viena de 23 de agosto de 1978, sobre sucesión de Estados en materia de Tratados, se ha de concluir que en el supuesto de separación de partes de un Estado para formar uno o más Estados, continúe o no en existencia el Estado predecesor, el principio dominante es el de continuidad de los tratados. Así se contiene en el artículo 34 de la Convención de Viena de 1978, en el que se establece para el supuesto de sucesión de Estados descrito, todo tratado que estuviera en vigor en la fecha de sucesión de Estados respecto la totalidad del territorio del Estado predecesor continuará en vigor respecto cada Estado sucesor así formado, estableciéndose como excepción en su apartado segundo a la aplicabilidad del tratado en cuestión el hecho de que los Estados interesados convinieren otra cosa, o que como consecuencia de la secesión de Estados, la aplicación del tratado fuera incompatible con su propio objeto y fin o cambiaran radicalmente las condiciones de su ejecución, debiendo concluirse que ninguna de las excepciones mencionadas concurren en el presente supuesto, por lo que ha de entenderse aplicable el Convenio entre el Reino de España y la Unión de Repúblicas Socialistas Soviéticas sobre asistencia judicial en materia civil, firmado en Madrid el 26 de octubre de 1990". Esta doctrina se reitera, entre otros, en el Auto del Tribunal Supremo (Sala de lo Civil) de 3 de julio de 2001 ${ }^{10}$ Auto del Tribunal Supremo (Sala de lo Civil) de 1 de julio de 2003 ${ }^{11}$ y Auto de la Audiencia Provincial de Madrid (Sección 24) de 14 de abril de 2011 ${ }^{12}$. Pero la omisión de este Convenio por parte del demandante es correcta, ya que su artículo 17.3.1) y 4) excluye expresamente de su ámbito de aplicación, en lo que se refiere al reconocimiento y ejecución de resoluciones judiciales, a las decisiones en materia concursal y a los laudos arbitrales. El Auto que ahora nos ocupa recuerda la existencia de este texto convencional, pero también de las exclusiones que hace su artículo 17 y por tanto no lo aplica.

6. La demanda tampoco invoca la Ley Concursal ${ }^{13}$ y en concreto sus artículos 220 a 226 , que regulan el reconocimiento de los procedimientos extranjeros de insolvencia. En este sentido hay que tener presente que el artículo 226 prevé el régimen de la eficacia en España de las medidas cautelares concursales dictadas en el extranjero. La actitud de la parte demandante es coherente con su posición de que solicitaba el exequatur de una resolución arbitral y por tanto ajena al marco de la Ley Concursal,

\footnotetext{
${ }^{7}$ BOE de 4 de octubre de 1975.

${ }^{8}$ BOE de 25 de junio de 1997.

${ }^{9} \mathrm{RJ} \backslash 1999 \backslash 1001$.

${ }^{10} \mathrm{RJ} \backslash 2001 \backslash 6521$.

${ }^{11} \mathrm{RJ} \backslash 2003 \backslash 6882$

12 JUR $\ 2011 \backslash 226796$.

${ }^{13}$ BOE de 10 de julio de 2003.
} 
puesto que los citados artículos se aplican a decisiones no sólo judiciales sino también administrativas, pero no a laudos arbitrales ${ }^{14}$.

7. En cuanto a las disposiciones legales alegadas en la demanda hay que comenzar diciendo que, como señala el propio Auto, el artículo 12.1 de la Ley de la Jurisdicción Voluntaria no es aplicable al caso, puesto que la pretensión del demandante versaba sobre un laudo arbitral dictado en un procedimiento de quiebra y no sobre un acto de jurisdicción voluntaria. Por el contrario, sí es adecuada la invocación del artículo 46 de la Ley Concursal en cuanto que regula el exequatur de los laudos extranjeros y que remite al Convenio de Nueva York de 10 de junio de 1958, cuyo artículo IV aduce el demandante. Finalmente este último también se remite al artículo 54 de la Ley de Cooperación Jurídica Internacional en Materia Civil, lo cual es correcto, si bien realmente debiera referirse genéricamente a los artículos que en la citada Ley regulan el reconocimiento y la ejecución de decisiones extranjeras y que operan en todo lo no previsto por el Convenio de Nueva York.

\section{Motivos por los que la Sala denegó el reconocimiento}

8. La Sala denegó el reconocimiento por dos motivos: la falta de presentación junto a la demanda del original o copia autenticada del convenio arbitral, tal como lo exige el artículo IV.1.b) del Convenio de Nueva York y que el arbitraje había recaído sobre una materia que en el ordenamiento español no es susceptible de resolverse mediante arbitraje.

9. Una reiteradísima jurisprudencia, cumpliendo el mandato del Convenio de Nueva York, ha sostenido que para obtener el exequatur es imprescindible que la demanda vaya acompañada de los documentos enumerados en el artículo IV de la mencionada Convención, y en concreto del original o copia autenticada del convenio arbitral. Así se afirma, por ejemplo, en los Autos del Tribunal Supremo de 1 de abril de $2003^{15}$ y 4 de marzo de $2003^{16}$, del Tribunal Superior de Justicia de Cataluña de 19 de septiembre de $2014{ }^{17}$ y de la Audiencia Provincial de Soria de 17 de septiembre de $2010^{18}$. Ciertamente, la jurisprudencia también ha formulado una interpretación que ella misma denomina "antiformalista", en el sentido de que entiende que aunque no se presente el convenio arbitral basta con probar la voluntad inequívoca de las partes de someterse a arbitraje, lo que se puede desprender de su propio comportamiento como cuando recurren el laudo, reconvienen en el procedimiento arbitral o desarrollan otras actividades similares ${ }^{19}$. En esta línea se pueden citar entre otros, además de los dos Autos del Tribunal Supremo a los que nos hemos referido más arriba, también el de 7 de octubre de $2003^{20}$ y los Autos de los Tribunales Superiores de Justicia de Andalucía de 14 de noviembre de $2013^{21}$ y de Madrid de 26 de septiembre de $2014^{22}$. En el caso que ahora nos ocupa, la parte demandante no presentó el convenio arbitral ni realizó el menor esfuerzo probatorio al respecto y tampoco tenemos datos sobre el comportamiento del demandado, por lo que, en principio, parece razonable la denegación del reconocimiento en base al

\footnotetext{
${ }^{14}$ M. Casado Abarquero, “Artículo 220”, en: P. Prendes Carril (Dir.), Tratado Práctico Concursal. Tomo IV Calificación del concurso. Conclusión y de la reapertura del concurso. Normas procesales generales y del sistema de recursos. Normas de Derecho Internacional Privado, Cizur Menor, 2009, pp. 890-891.

${ }^{15}$ ECLI:ES:TS:2003:3649A.

16 ECLI:ES:TS:2003:2447A.

17 ECLI:ES:TSJCAT:2014:307A.

${ }^{18}$ ECLI:ES:APSO:2010:132A.

${ }^{19}$ Esta interpretación antiformalista iría en línea con la Recomendación de la UNCITRAL de 7 de julio de 2006 cuando dice: "1. Recomienda que el párrafo 2) del artículo II, de la Convención sobre el Reconocimiento y la Ejecución de las Sentencias Arbitrales Extranjeras, hecha en Nueva York el 10 de junio de 1958, se aplique reconociendo que las circunstancias que describe no son exhaustivas". https://uncitral.un.org/es/texts/arbitration/explanatorytexts/recommendations/foreign_arbitral_awards

${ }^{20}$ ECLI:ES:TS:2003:10137A.

${ }^{21}$ ECLI:ES:TSJAND:2013:54A.

${ }^{22}$ ECLI:ES:TSJM:2014:57A.
} 
incumplimiento del artículo IV.1.b) del Convenio de Nueva York. No obstante, no podemos olvidar que, como ya hemos apuntado antes, tal vez el procedimiento seguido en Rusia no era propiamente arbitral.

10. El artículo V.2.a) del Convenio de Nueva York establece como causa apreciable de oficio para denegar el reconocimiento y la ejecución de un laudo arbitral extranjero, el que según el ordenamiento del Estado requerido el objeto de la diferencia no sea susceptible de solucionarse por vía de arbitraje $^{23}$. En nuestro sistema jurídico el artículo 2.1 de la Ley de Arbitraje establece que "Son susceptibles de arbitraje las controversias sobre materias de libre disposición conforme a derecho". Los procedimientos concursales no son de libre disposición, por consiguiente no cabe reconocer en España un laudo referente a dicha materia. Así se aprecia correctamente en la decisión ahora comentada.

\section{Un problema subyacente: el reconocimiento y ejecución de medidas cautelares adoptadas por árbitros en el extranjero}

11. El Auto sobre el que ahora trabajamos rechazó el exequatur por los motivos que hemos visto en el apartado anterior y no entró en un arduo problema previo que es el del reconocimiento y ejecución en España de las medidas cautelares adoptadas por árbitros en el extranjero ${ }^{24}$. A este respecto hay que tener en cuenta que se trataba de una medida decidida por los árbitros, al parecer en el seno del procedimiento y a solicitud de una de las partes.

12. En un primer momento, la cuestión del arbitraje internacional y la justicia cautelar gravitó fundamentalmente sobre la cooperación de los órganos judiciales en el procedimiento arbitral, es decir, se trataba de medidas cautelares adoptadas por los jueces estatales en relación con un arbitraje. De esta manera, como eran decisiones judiciales en principio era admisible su exequatur, si bien en la práctica no siempre era fácil obtenerlo ${ }^{25}$. No obstante, pese a algunas dudas o reticencias ${ }^{26}$, en la actualidad es muy aceptado que los árbitros puedan tomar medidas provisionales o cautelares en el arbitraje internacional y en esta dirección se orientan los textos internacionale ${ }^{27}$, los Reglamentos arbitrales ${ }^{28}$ y la consolidación de figuras como la del árbitro de emergencia ${ }^{29}$. Ante esta realidad se plantea el problema del

${ }^{23}$ Vid. el ya citado Auto del Tribunal Supremo de 1 de abril de 2003 y el del Tribunal Superior de Justicia de Madrid de 2 de noviembre de 2017 (ECLI:ES:TSJM:2017:494A).

${ }^{24}$ Un análisis reciente y muy completo de este problema lo encontramos en: X. OrbegGozo MiguEL, Justicia cautelar y UE: una perspectiva mixta (jurisdiccional y arbitral) y su proyección sobre el ámbito marítimo, Universidad del País Vasco-Euskal Herriko Unibertsitatea, 2019, especialmente pp. 197-227.

${ }^{25}$ A. L., Calvo Caravaca, "Medidas cautelares y arbitraje privado internacional", Diario La Ley, n ${ }^{\circ}$ 6128, 16 de noviembre de 2004. A. L., Calvo Caravaca, J. Carrascosa González, "Medidas cautelares y comercio internacional", Cuadernos de Derecho Judicial, no 18, 2004, pp. 409 y ss. J. C. FERnÁNDEZ RozAs, “Arbitraje y justicia cautelar”, Revista de la Corte Española de Arbitraje, vol. XXII, 2007, pp. 23-60. M. V. SÁnchez Pos, "Del juez al árbitro de emergencia. El impacto de la Ley Modelo en la progresiva atribución de la tutela cautelar a los árbitros en la legislación cautelar española y en otras legislaciones estatales", Arbitraje, vol. XI, n 2, 2018, pp. 403-442. C. BROwn, "The Enforcement of Interim Measures Ordered by Tribunals and Emergency Arbitrators in International Arbitration", en: A. VAN DEN BERG (ed.), International arbitration, the coming of a new age, Alpheen aam den Rijn, 2013, pp. 279-291. I. M. SchwARTZ, "Interim and emergency relief in arbitration proceedings", Dispute Resolution Journal, febrero-abril, 2008, pp. 56-62.

${ }^{26}$ G. B. Born, International Arbitration, Cases and Materials, $2^{\mathrm{a}}$ ed., Alpheen aam den Rijn, 2015, p. 875. H. VAN HoutTe, "Ten Reasons Against a Proposal for Ex Parte Interim Measures of Protection in Arbitration”, Arbitration International, vol. 20, no 1, 2004, pp. 85-96.

${ }_{27}$ Paradigmático es el artículo 17 de la Ley Modelo de la UNCITRAL sobre Arbitraje Comercial Internacional. https:// uncitral.un.org/sites/uncitral.un.org/files/media-documents/uncitral/es/07-87001_ebook.pdf

${ }^{28}$ Por ejemplo: artículos 28 y 29 del Reglamento de Arbitraje CCI, 9B y 25 del Reglamento de la Corte de Arbitraje Internacional de Londres y 6 y 24 del Reglamento de Mediación y de Arbitraje del Centro Internacional para la Resolución de Disputas. https://iccwbo.org/content/uploads/sites/3/2017/03/ICC-2017-Arbitration-and-2014-Mediation-Rules-spanish-version.pdf https://www.lcia.org/Dispute_Resolution_Services/lcia-arbitration-rules-2014.aspx

https://www.icdr.org/sites/default/files/document_repository/ICDR_Rules.pdf

29 E. Fernández Masiá, "La irrupción del árbitro de emergencia en el arbitraje comercial internacional”, Cuadernos de Derecho Transnacional, vol. 9, № 1, 2017, pp. 82-98. 
reconocimiento y ejecución de las decisiones cautelares dictadas por los árbitros en aquellos supuestos en los que las partes litigantes se niegan a cumplirlas voluntariamente.

13. En el ordenamiento español no disponemos de ninguna norma que responda claramente a esta cuestión. Ésta es una importante carencia que debe ser prontamente corregida por el Legislador. En un primer momento hay que plantearse si el Convenio de Nueva York se puede aplicar al exequatur de medidas cautelares adoptadas por árbitros ${ }^{30}$. A este respecto la clave radica en la interpretación que se haga de los términos de su artículo V.1.e), que establece que es motivo para denegar el reconocimiento y la ejecución a instancia de parte que "la sentencia no es aún obligatoria para las partes". Hay autores que entienden que este tipo de medidas no tienen carácter definitivo, puesto que pueden ser modificadas o revocadas, de lo que concluyen que no tienen la nota de obligatoriedad a la que se refiere el citado artículo y por tanto no pueden ser reconocidas ni ejecutadas en el extranjero. A esto habría que añadir que el texto del Convenio no hace ninguna referencia expresa a las medidas cautelares y la mayor parte de la jurisprudencia tradicionalmente ha sostenido esta misma interpretación ${ }^{31}$.

14. Sin embargo, también cabe otra interpretación más avanzada consistente en sostener que lo determinante no es que la decisión arbitral sea definitiva sino que sea vinculante para las partes. De esta manera si el laudo de medidas cautelares es susceptible de ejecución en el lugar en el que se ha dictado, está vigente en el momento en el que se solicita el exequatur, vincula a los litigantes y ha respetado los derechos procesales de la parte a la que se ha impuesto la medida, cabría conceder su reconocimiento y ejecución ${ }^{32}$. Esta posición tiene reflejo en alguna jurisprudencia comparada ${ }^{33}$. En este sentido hay que recordar que la jurisprudencia española ha dicho reiteradamente que no se puede confundir laudo obligatorio con firmeza del laudo; ciertamente lo ha dicho en casos en los que se pretendía el exequatur de laudos recurridos ante los Tribunales del país en el que se habían dictado, pero en esencia esta doctrina sirve para apuntalar la interpretación a la que nos referimos. Por ejemplo en el reciente Auto del Tribunal Superior de Justicia de Murcia de 12 de abril de $2019^{34}$ se dice: "No habiendo sido anulado ni suspendido el laudo, éste era obligatorio para las partes al margen de que se hubieran o no intentado acciones de cualquier tipo tendentes a su anulación. No debe confundirse laudo obligatorio con firmeza del laudo (ATS 10 febrero 2002 y 10 diciembre 2002). Así resulta en primer lugar, ...del artículo 34-6 del Reglamento de la Corte Internacional de Arbitraje, al que las partes se sometieron ... cuando señala que: "Todo laudo es obligatorio para las partes. Al someter su controversia a arbitraje según el Reglamento, las partes se obligan a cumplir sin demora cualquier laudo que se dicte y se considerará que han renunciado a cualesquiera vías de recurso a las que puedan renunciar válidamente”. [...] La obligatoriedad del aludo resulta también de lo previsto en el artículo 45.1 de la Ley española de Arbitraje, cuando opta por su ejecutabilidad aun cuando contra él se haya ejercitado acción de anulación, salvo acuerdo de suspensión por el tribunal encargado de la ejecución y previa prestación de la oportuna caución”.

15. A esto habría que añadir el espíritu y finalidad que anima al Convenio de Nueva York, que son justamente los de favorecer la libre circulación de decisiones arbitrales. Y por otro lado, hay que re-

\footnotetext{
${ }^{30}$ X. Orbegozo Miguel, Justicia cautelar y UE ..., pp. 217 y ss.

${ }^{31}$ C. Brown, "The Enforcement ...”, p. 286. K. P. Berger, Private Dispute Resolution in International Business. Negotiation, Mediation, Arbitration, $3^{\mathrm{a}}$ ed., Alpheen aam den Rijn, 2015, p. 453. A. YeșiLIRMAK, Provisional measures in international comercial arbitration, Alpheen aam den Rijn, 2005, pp. 259-261. M. Gómez JenE, “Arbitraje internacional y Anteproyecto de Ley de Cooperación Jurídica Internacional en Materia Civil”, La Ley, no 8388, 30 de septiembre de 2014, p. 6.

${ }^{32}$ M. M. Verdías Mezzera, "Legge Ferenda. La necesidad de dar efecto útil -y formal- a las órdenes emitidas por "árbitros de emergencia”"', Arbitraje, vol. XII, no 1, 2019, p. 194. P. Simsive, "Indirect Enforceability of Emergency Arbitrator's Orders", Kluwer Arbitration Blog, 15 de abril de 2015. http://arbitrationblog.kluwerarbitration.com/2015/04/15/indirect-enforceabilityof-emergency-arbitrators-orders/

33 Véanse las resoluciones citadas en: X. Orbegozo Miguel, Justicia cautelar y UE ..., pp. 218 y 223.

${ }^{34}$ ECLI:ES:TSJMU:2019:12A. M. J. CASTELlanos Ruiz, "Exequatur de laudos arbitrales extranjeros en España: comentario al Auto del TSJ de Murcia de 12 de abril de 2019", Cuadernos de Derecho Transnacional, vol. 12, No 1, 2020, pp. 512-530.

En la misma línea se pueden citar los Autos del Tribunal Superior de Justicia de Cataluña de 17 de noviembre de 2011 (ECLI:ES:TSJCAT:2011:525A) y 15 de mayo de 2014 (ECLI:ES:TSJCAT:2014:184A).
} 
cordar la razón de ser de las medidas cautelares, que es garantizar o asegurar el cumplimiento del laudo final. Este objetivo sólo se puede alcanzar en el ámbito arbitral si se propicia el exequatur de dichas medidas cuando son dictadas por los árbitros. En este sentido no deja de ser curioso, o tal vez significativo, que en el caso ahora analizado, la Sala aplicó el Convenio de Nueva York al reconocimiento y ejecución de la medida cautelar arbitral dictada en Rusia sin hacer ninguna precisión o justificación al respecto y simplemente en base a la remisión que el artículo 46.2 de la Ley de Arbitraje realiza al citado Convenio. Parecería que daba por supuesto que su aplicación a las medidas cautelares era indiscutible.

16. En este mismo orden de cosas, desde la perspectiva del Derecho español de origen interno hay que reparar en el artículo 41.4 de la Ley de Cooperación Jurídica Internacional en Materia Civil, que admite el reconocimiento y ejecución de medidas cautelares y provisionales extranjeras, cuando su denegación implique una vulneración del derecho a la tutela judicial efectiva y siempre que se hubiesen adoptado respetando los derechos procesales, fundamentalmente el derecho a ser oído, de la parte contraria ${ }^{35}$.

\section{Conclusiones}

17. Estamos ante un caso extraño, como es el de la solicitud del exequatur de una medida cautelar dictada por un tribunal arbitral en un procedimiento concursal. Posiblemente la decisión extranjera no partió de un auténtico arbitraje. La Sala denegó el reconocimiento por dos motivos, como son la falta de presentación del convenio arbitral y que la decisión había recaído sobre una materia no susceptible de resolverse por arbitraje en el Derecho español; ambos motivos son perfectamente admisibles e indudablemente concurrían en el supuesto. El Auto no entra en el problema del exequatur de las medidas cautelares dictadas por árbitros en el extranjero, pero en él se aplica el Convenio de Nueva York como si se diese por supuesto que era la norma competente y sin construir ninguna justificación al respecto.

${ }^{35}$ E. FeRnÁNDEZ Masí́, "La irrupción del árbitro ...,", p. 95. 